\title{
Relationship of Soluble RAGE with Insulin Resistance and Beta Cell Function during Development of Type 2 Diabetes Mellitus
}

\author{
Subrata Kumar Biswas, ${ }^{1}$ Sabreena Mohtarin, ${ }^{1}$ Sonchita Rani Mudi, ${ }^{2}$ Taznuva Anwar, ${ }^{1}$ \\ Laila Anjuman Banu, ${ }^{3}$ Sheikh Md. Khorshed Alam, ${ }^{1}$ Md. Fariduddin, ${ }^{4}$ and M. Iqbal Arslan ${ }^{1}$ \\ ${ }^{1}$ Department of Biochemistry, Bangabandhu Sheikh Mujib Medical University (BSMMU), Shahbag, Dhaka 1000, Bangladesh \\ ${ }^{2}$ Department of Biochemistry and Cell Biology, Bangladesh Institute of Research and Rehabilitation in Diabetes, \\ Endocrine and Metabolic Disorders (BIRDEM), 122 Kazi Nazrul Islam Avenue, Shahbag, Dhaka 1000, Bangladesh \\ ${ }^{3}$ Department of Anatomy, Bangabandhu Sheikh Mujib Medical University (BSMMU), Shahbag, Dhaka 1000, Bangladesh \\ ${ }^{4}$ Department of Endocrinology, Bangabandhu Sheikh Mujib Medical University (BSMMU), Shahbag, Dhaka 1000, Bangladesh
}

Correspondence should be addressed to Subrata Kumar Biswas; su.biswas@yahoo.com

Received 14 April 2015; Accepted 12 May 2015

Academic Editor: Roberto Mallone

Copyright (C) 2015 Subrata Kumar Biswas et al. This is an open access article distributed under the Creative Commons Attribution License, which permits unrestricted use, distribution, and reproduction in any medium, provided the original work is properly cited.

\begin{abstract}
This study examined whether circulating levels of soluble receptor for advanced glycation end products (sRAGE) alter in prediabetes and correlate with insulin resistance (IR) and beta cell function in prediabetes and newly diagnosed type 2 diabetes mellitus (T2DM). Subjects without previous history of diabetes were recruited and grouped as control, prediabetes, and newly diagnosed T2DM. The control subjects $(n=40)$ and people with prediabetes $(n=52)$ and diabetes $(n=66)$ were similar in terms of age, sex, BMI, systolic and diastolic BP, and fasting insulin level. HOMA-IR was found significantly higher in people with diabetes than control subjects $(p<0.001)$ and people with prediabetes $(p=0.005)$; and HOMA-\%B was found significantly deteriorated in people with diabetes $(p<0.001)$ compared to control subjects and people with prediabetes. However, serum sRAGE levels did not show any significant alteration in people with prediabetes compared to control subjects. Moreover, univariate and multivariate analyses did not identify any significant correlation and statistical association of sRAGE with HOMA-IR and HOMA-\%B in people with prediabetes and newly diagnosed T2DM. Our data suggest that serum sRAGE levels do not alter in people with prediabetes compared to control subjects and do not correlate or associate with IR and beta cell function during development of T2DM.
\end{abstract}

\section{Introduction}

The receptor for advanced glycation end products (RAGE) is a cell surface receptor of immunoglobulin superfamily. RAGE activation through ligand binding can induce chronic inflammation and oxidative stress, and it has been linked with diseases like diabetic complications, cardiovascular and neurodegenerative diseases, and cancer [1]. A soluble form of RAGE (sRAGE), which is a splice variant of full-length RAGE or a shedding/cleavage product of membrane-bound RAGE, has been found circulating in the plasma $[2,3]$. The sRAGE can bind and sequester RAGE ligands and thereby can reduce RAGE activation. Therefore, the sRAGE is generally considered as protective against diseases originating from RAGE activation [1-3].
RAGE-ligand interaction was previously claimed to be involved in the pathogenesis of autoimmune diabetes, and treatment with sRAGE was shown to effectively prevent transfer of diabetes into $\mathrm{NOD} /$ scid mice that receive spleen cells from a diabetic NOD donor [4]. Subsequently, blockade of high-mobility group box 1, a RAGE ligand, was shown to inhibit insulitis progression and diabetes development in NOD mice [5]. A minor role of genetic variation in RAGE was also suggested to be associated with insulin resistance (IR) in a human population [6]. However, recent studies have suggested that low levels of circulating sRAGE may be involved in the development of diabetes mellitus [7-9]. A declining level of sRAGE at the time of seroconversion to autoantibody positivity has been suspected to be a predictor of type 1 diabetes $[7,8]$, and an independent association has 
been found between low levels of sRAGE and development of type 2 diabetes mellitus (T2DM) [9]. However, the relationship of sRAGE with the underlying pathophysiological mechanisms of T2DM has not been specifically explored.

The IR and beta cell dysfunction are two core defects of $\mathrm{T} 2 \mathrm{DM}$, and the "prediabetes" is a category of increased risk of developing T2DM in subjects who have not yet fulfilled the criteria to be diabetic [10]. To explore the involvement or participation of sRAGE in the development of T2DM, present study was designed to assess whether sRAGE levels alter in prediabetes and correlate with IR and beta cell function in prediabetes and newly diagnosed T2DM.

\section{Subjects and Methods}

A total of 158 participants were recruited from those who came for diabetes screening at the Bangabandhu Sheikh Mujib Medical University, Dhaka, Bangladesh, after giving written consent. This cross-sectional study was conducted according to the Declaration of Helsinki and was approved by the institutional ethical review committee. Participants were grouped as control (normoglycemic), prediabetes, and newly diagnosed T2DM based on their blood glucose (fasting and $2 \mathrm{hrs}$ after 75 grams glucose load) and HbAlc levels. As recommended by American Diabetic Association (ADA) [10], diabetes was considered with a fasting glucose level $\geq 7.0 \mathrm{mmol} / \mathrm{L}$ and/or $2 \mathrm{hrs}$ blood glucose $\geq 11.1 \mathrm{mmol} / \mathrm{L}$ and/or $\mathrm{HbAlc} \geq 6.5 \%$; and prediabetes was considered with a fasting glucose level 5.6-6.9 mmol/L (impaired fasting glucose, IFG) and/or $2 \mathrm{hrs}$ blood glucose 7.8-11.0 mmol/L (impaired glucose tolerance, IGT) and/or HbAlc 5.7-6.4\%. Control subjects did not qualify for any of the above glycemic or HbAlc criteria. Subjects with previous history of diabetes and those suffering from hypertension, chronic liver and kidney diseases, or any other acute/chronic inflammatory conditions as well as pregnant and lactating women and regular drug users were excluded. A detailed medical history was taken and clinical examination including height, weight, and blood pressure data were recorded for all subjects.

A fasting blood sample was collected after an overnight fasting of $>8$ hours and a second blood sample was collected 2 hours after 75 grams glucose load on the same day from all subjects. Fasting and 2 hours after glucose levels were measured by enzymatic spectrophotometric method using Dimension RxL Max clinical chemistry analyzer (Siemens Healthcare Diagnostics Inc., Newark, DE, USA). HbAlc levels were measured by ion-exchange high-performance liquid chromatography in a Bio-Rad D-10 instrument (Bio-Rad Laboratories Inc., Hercules, CA, USA). Fasting serum insulin levels were measured by microparticle enzyme immunoassay technique (Abbott Diagnostics, Wiesbaden, Germany) using an Abbott AxSYM system with an interassay coefficient of variation $(\mathrm{CV})<5 \%$. Fasting serum sRAGE levels were measured by ELISA in triplicate, as suggested by the manufacturer (R\&D Systems, Minneapolis, MN, USA) with an interassay $\mathrm{CV}$ of $<7 \%$. The IR and beta cell function were calculated as homeostasis model assessment of IR (HOMA-IR) [(glucose $x$ insulin)/22.5] and homeostasis model assessment of beta cell function $(\mathrm{HOMA}-\% \mathrm{~B})[(20 \times$ insulin $) /($ glucose -3.5$)]$, respectively, where glucose was in $\mathrm{mmol} / \mathrm{L}$ and insulin was in $\mu \mathrm{U} / \mathrm{mL}$.

Statistical Analysis. All statistical analyses were performed using SPSS version 20.0 (SPSS Inc., Chicago, IL). Data are presented as mean \pm SD. Variables with a skewed distribution are expressed as median (interquartile range) and were log transformed before statistical analysis. Comparison among multiple groups was done by one-way ANOVA followed by Bonferroni corrected $t$-test. Categorical variables were analyzed by $\chi^{2}$ test. Bivariate correlations were determined by Pearson's univariate correlation analysis. Stepwise multivariate linear regression models were calculated to demonstrate independent relationships of sRAGE and other variables with HOMA-IR and HOMA-\%B. The HOMA-IR was used as dependent variable in one model and the HOMA- $\% \mathrm{~B}$ as dependent variable in another model with the following independent variables: age, sex, BMI, systolic and diastolic BP, glucose 2 hours, HbAlc, sRAGE, and HOMA-IR/HOMA$\%$ B. A $p$ value of $\leq 0.05$ for $F$-values was taken as criterion for entering variables in the model and $p \geq 0.1$ for $F$-values was taken as criterion for exclusion of variables from the model. Fasting glucose and insulin levels were not included in the models since they were directly used for calculation of HOMA-IR and HOMA-\%B. A two-tailed value of $p<0.05$ was considered statistically significant.

\section{Results}

As shown in Table 1 , the age ( $40.2 \pm 8.7 ; 20-58$ years) and sex $(\mathrm{m}=73, \mathrm{f}=85)$ distributions of the 158 study participants were found similar among control subjects $(n=40)$ and people with prediabetes $(n=52)$ and diabetes $(n=66)$. The BMI, systolic and diastolic BP, and fasting insulin levels were also found similar among the three groups. But the fasting and 2 hours blood glucose and HbAlc levels were found significantly $(p<0.001)$ elevated in people with diabetes compared to control subjects and people with prediabetes. HOMA-IR was found significantly higher in people with diabetes than control subjects $(p<0.001)$ and people with prediabetes $(p=0.005)$. As expected, HOMA-\%B was found markedly decreased in people with diabetes $(p<0.001)$ compared to control subjects and people with prediabetes.

However, as shown in Figure 1, serum sRAGE levels in people with prediabetes $(656,463-968$; median, interquartile range in $\mathrm{pg} / \mathrm{mL}$ ) did not show any significant difference compared with that of control subjects $(626,413-864)$ and people with diabetes $(646,493-817)$.

We next investigated the relationship of sRAGE with markers of IR and beta cell function by using Pearson's correlation test. The sRAGE level did not show any significant correlation with HOMA-IR in all the study subjects $(r=$ $0.007, p=0.94)$, in people with diabetes $(r=-0.07, p=$ $0.64)$ or prediabetes $(r=0.22, p=0.17)$. Similarly, sRAGE level did not show any significant correlation with HOMA$\% \mathrm{~B}$ in all the study subjects $(r=-0.02, p=0.87)$, in people with diabetes $(r=0.04, p=0.80)$ or prediabetes $(r=0.24, p=0.12)$ (data not shown in the table). For further statistical analysis, we merged the people with 
TABLE 1: Clinical and biochemical characteristics of the study subjects.

\begin{tabular}{|c|c|c|c|}
\hline Variables & Control subjects $(n=40)$ & People with prediabetes $(n=52)$ & People with diabetes $(n=66)$ \\
\hline Age (years) & $38.4 \pm 7.6$ & $38.9 \pm 9.5$ & $42.5 \pm 8.4$ \\
\hline Sex, male/female $(n)$ & $18 / 22$ & $24 / 28$ & $31 / 35$ \\
\hline BMI $\left(\mathrm{kg} / \mathrm{m}^{2}\right)$ & $25.9 \pm 6.2$ & $27.2 \pm 5.5$ & $25.4 \pm 4.6$ \\
\hline $\mathrm{SBP}(\mathrm{mm} \mathrm{Hg})$ & $116.3 \pm 8.5$ & $118.1 \pm 8.6$ & $119.3 \pm 8.3$ \\
\hline $\mathrm{DBP}(\mathrm{mm} \mathrm{Hg})$ & $74.7 \pm 8.4$ & $76.1 \pm 7.8$ & $75.4 \pm 8.2$ \\
\hline Glucose, fasting $(\mathrm{mmol} / \mathrm{L})$ & $4.7 \pm 0.5$ & $5.1 \pm 0.6$ & $9.7 \pm 3.6^{\mathrm{a}}$ \\
\hline Glucose, 2 hrs (mmol/L) & $6.5 \pm 0.9$ & $8.2 \pm 1.4$ & $16.3 \pm 5.6^{\mathrm{a}}$ \\
\hline $\operatorname{HbAlc}(\%)$ & $5.0 \pm 0.4$ & $5.7 \pm 0.5$ & $8.1 \pm 2.4^{\mathrm{a}}$ \\
\hline $\mathrm{HbAlc}(\mathrm{mmol} / \mathrm{mol})$ & $30.9 \pm 4.3$ & $38.4 \pm 5.4$ & $65.5 \pm 26.0^{\mathrm{a}}$ \\
\hline Insulin* $(\mu \mathrm{U} / \mathrm{mL})$ & $9.3(6.7-14.8)$ & $11.5(8.6-17.8)$ & $9.4(5.9-18.6)$ \\
\hline HOMA-IR* & $2.0(1.6-3.1)$ & $2.6(1.9-4.1)$ & $4.1(2.7-7.7)^{\mathrm{bc}}$ \\
\hline HOMA-\%B* $(\%)$ & $168(95-313)$ & $149(109-230)$ & $39(19-89)^{\mathrm{a}}$ \\
\hline
\end{tabular}

Data are mean \pm SD, number, and median (interquartile range). ${ }^{*}$ Log-transformed variables, values given are median (interquartile range). ${ }^{a} p<0.001$ versus control subjects and people with prediabetes; ${ }^{\mathrm{b}} p<0.001$ versus control subjects; ${ }^{\mathrm{c}} p=0.005$ versus people with prediabetes. BMI, body mass index; SBP, systolic blood pressure; DBP, diastolic blood pressure; HOMA-IR, homeostasis model assessment of insulin resistance; HOMA-\%B, homeostasis model assessment of beta cell function.

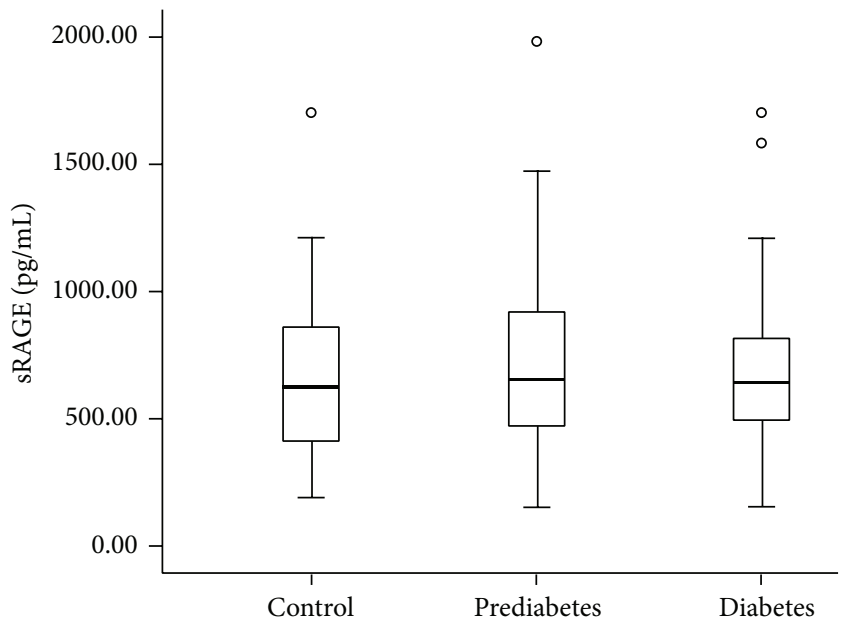

FIGURE 1: Box plot showing the sRAGE concentrations in control subjects and in people with prediabetes and newly diagnosed type 2 diabetes. The line within the boxes represents the median, the bottom of each box represents the 25th percentile, and the top of the box represents the 75th percentile. The whiskers represent the 5 th and 95th percentiles, and the small circles represent outliers.

prediabetes and newly diagnosed type 2 diabetes together (PD + DM group, $n=118$ ) considering that both groups have similar underlying pathophysiological defects responsible for glucose intolerance and hyperglycemia. Characteristics of the participants of this PD + DM group as well as relationship of HOMA-IR and HOMA-\%B with other variables were shown in Table 2. Of note, sRAGE levels did not show any significant correlation with HOMA-IR and HOMA-\%B even in the participants of this PD + DM group (Table 2). But HOMAIR showed marginal correlation with 2 hours glucose levels $(r=0.20, p=0.05)$ and significant correlation with HOMA$\% \mathrm{~B}(r=0.27, p=0.007)$, and HOMA-\%B showed significant correlation with 2 hours glucose levels $(r=0.70, p<0.001)$ and HbAlc $(r=0.72, p<0.001)$ (Table 2$)$.
A stepwise multivariate linear regression model with HOMA-IR as dependent variable and age, sex, BMI, systolic and diastolic BP, 2 hours glucose level, HbAlc, sRAGE, and HOMA- $\% \mathrm{~B}$ as independent variables showed independent association of HOMA-IR with BMI $(\beta=0.21, p=0.03)$, 2 hours glucose levels ( $\beta=0.67, p<0.001$ ), and HOMA$\% \mathrm{~B}(\beta=0.74, p<0.001)$ in $\mathrm{PD}+\mathrm{DM}$ group $\left(R^{2}=0.405\right)$ (Table 2).

Another model with HOMA-\%B as dependent variable and age, sex, BMI, systolic and diastolic BP, 2 hours glucose level, HbAlc, sRAGE, and HOMA-IR as independent variables showed independent association of HOMA- $\% \mathrm{~B}$ with 2 hours glucose level $(\beta=0.33, p=0.028), \operatorname{HbAlc}(\beta=0.41$, $p=0.005)$, and HOMA-IR $(\beta=0.46, p<0.001)$ in PD + DM group $\left(R^{2}=0.629\right)$ (Table 2). Furthermore, the above regression models when applied separately for the people with prediabetes (not shown in the table) showed significant association of HOMA-IR with BMI $(\beta=0.33, p=0.006)$ and HOMA-\%B $(\beta=0.62, p<0.001)\left(R^{2}=0.530\right)$ and HOMA$\% \mathrm{~B}$ only with HOMA-IR $\left(\beta=0.67, p<0.001, R^{2}=0.433\right)$. Such models when applied for the people with diabetes (not shown in the table) showed significant association of HOMAIR with HbAlc $(\beta=0.43, p=0.009)$ and HOMA-\%B $(\beta=0.92, p<0.001)\left(R^{2}=0.536\right)$ and HOMA-\%B with HbAlc $(\beta=-0.52, p<0.001)$ and HOMA-IR $(\beta=0.61$, $p<0.001)\left(R^{2}=0.694\right)$. But none of the above models showed any significant association of sRAGE with HOMAIR and HOMA-\%B in people with prediabetes and newly diagnosed T2DM.

\section{Discussion}

The T2DM develops insidiously with gradual impairment of glucose tolerance due to IR and beta cell dysfunction [10]. Before development of overt diabetes mellitus there is a state of prediabetes characterized by IGT and/or IFG. People with prediabetes suffer from increased risk of developing T2DM 
TABLE 2: Patient characteristics and associations of HOMA-IR and HOMA-\%B with different variables in people with prediabetes and newly diagnosed type 2 diabetes.

\begin{tabular}{|c|c|c|c|c|c|}
\hline & \multirow{2}{*}{ Characteristics PD + DM $(n=118)$} & \multicolumn{2}{|c|}{ HOMA-IR ${ }^{*}$} & \multicolumn{2}{|c|}{ HOMA-\%B* } \\
\hline & & Univariate $(r)$ & Multivariate $(\beta)$ & Univariate $(r)$ & Multivariate $(\beta)$ \\
\hline Age (years) & $40.8 \pm 9.0$ & 0.06 & - & -0.06 & - \\
\hline Sex, $\mathrm{m} / \mathrm{f}(n)$ & $55 / 63$ & - & - & - & - \\
\hline $\operatorname{BMI}\left(\mathrm{Kg} / \mathrm{m}^{2}\right)$ & $26.17 \pm 5.03$ & 0.12 & $0.21^{\mathrm{a}}$ & 0.19 & - \\
\hline SBP (mm Hg) & $118.8 \pm 8.4$ & -0.03 & - & -0.12 & - \\
\hline DBP (mm Hg) & $75.7 \pm 8.0$ & -0.09 & - & -0.13 & - \\
\hline Glucose, 2 hrs (mmol/L) & $12.8 \pm 5.9$ & $0.20^{\mathrm{a}}$ & $0.67^{\mathrm{c}}$ & $-0.70^{c}$ & $-0.33^{\mathrm{a}}$ \\
\hline $\operatorname{HbAlc}(\%)$ & $6.9 \pm 2.1$ & 0.06 & - & $-0.72^{\mathrm{c}}$ & $-0.41^{\mathrm{b}}$ \\
\hline HOMA-IR* & $3.2(2.3-5.3)$ & - & - & $0.27^{\mathrm{b}}$ & $0.46^{c}$ \\
\hline HOMA-\%B* & $95(33-163)$ & $0.27^{\mathrm{b}}$ & $0.74^{\mathrm{c}}$ & - & - \\
\hline $\operatorname{sRAGE}(\mathrm{pg} / \mathrm{mL})^{*}$ & $646(482-897)$ & 0.04 & - & 0.08 & - \\
\hline
\end{tabular}

Data are mean $\pm \mathrm{SD}$, number, and median (interquartile range). ${ }^{*}$ Values were not normally distributed and log-transformed for statistical analysis. $r$, Pearson's univariate correlation coefficient; $\beta$, standardized coefficient as given by stepwise multivariate regression analysis. Dashes in the multivariate columns correspond to variables excluded from the model due to $p \geq 0.1$ for $F$-values. PD + DM, people with prediabetes and diabetes together; BMI, body mass index; SBP, systolic blood pressure; DBP, diastolic blood pressure; ${ }^{\mathrm{a}} p \leq 0.05 ;{ }^{\mathrm{b}} p<0.01 ;{ }^{\mathrm{c}} p<0.001$.

in near future compared with people with normal glucose tolerance [10]. In the present study we investigated the sRAGE levels in prediabetes and the relationship of sRAGE with IR and beta cell function in people with prediabetes and newly diagnosed T2DM. We found that sRAGE levels do not alter in people with prediabetes compared with normoglycemic control subjects. Moreover, we did not observe any correlation or statistical association of sRAGE with IR and beta cell function in people with prediabetes and newly diagnosed T2DM.

Discrepancy exists regarding the sRAGE levels in diabetes-higher, lower, and even similar levels of sRAGE have been reported in people with T2DM compared with control subjects without diabetes (reviewed in [11]). The reason for this discrepancy among studies is not clear but the presence of confounding variables like the duration of diabetes, presence of hypertension and use of antihypertensive drugs, smoking habit, and chronic kidney and inflammatory diseases may contribute to this $[11,12]$. It has been shown that longer duration of diabetes is associated with increased advanced glycation end products (AGE) generation and AGE-stimulated increased RAGE expression. The increased RAGE expression in turn may increase sRAGE level by shedding of membrane-bound RAGE [11]. If so, people with prediabetes and newly-diagnosed T2DM may not show a significant alteration in sRAGE level since they may not have experienced a heavy load of AGE yet. In fact, a large study recently found no difference in sRAGE levels between children with newly diagnosed type 1 diabetes and control subjects and emphasized the importance of evaluating sRAGE levels in children with prediabetes [13]. But, to our knowledge, the sRAGE status in people with prediabetes was unknown until recently. During the preparation of this paper, Di Pino et al. published cardiovascular risk profile in prediabetes and type 2 diabetes, where they found similar levels of sRAGE in control subjects and in people with prediabetes and new-onset T2DM [14] as we found in the present study. However, it should be noted that $68 \%$ of the people with prediabetes of the study by Di Pino et al. were normal glucose tolerant (without having IFG or IGT) since Di Pino et al. grouped the study subjects only on the basis of HbAlc. Thus it was uncertain whether the findings of Di Pino et al. would be equally valid for people with prediabetes defined by standard ADA criteria (IFG and/or IGT and/or HbAlc 5.7-6.4\%) [10]. In the present study, we significantly added to the findings of Di Pino et al. by showing that sRAGE levels do not alter in people with prediabetes, defined by standard ADA criteria, compared with control subjects and people with newly diagnosed T2DM.

The IR and beta cell dysfunction are two core defects that are found in variable extent in people with T2DM [10]. However, the relationship of sRAGE with IR and beta cell function was so far not clear. To our knowledge, Basta et al. previously found negative correlation between sRAGE level and IR taken control subjects and people with diabetes together in the analysis [15]. But this relationship disappeared when they analyzed the data in age-selected control subjects and people with diabetes separately. Furthermore, an independent negative association of sRAGE with IR also disappeared when they performed multivariate regression analyses on control subjects and people with diabetes separately [15]. In fact, the people with diabetes of the study by Basta et al. [15] were significantly different from control subjects in respect to age, number of hypertensive subjects, and use of antihypertensive drugs, factors that are known to affect sRAGE levels [11]. Taken together, the negative relationship between sRAGE and IR shown by Basta et al. was questionable. In the present study in a relatively homogenous set of study subjects we found that sRAGE levels do not correlate and do not show any association with IR in people with prediabetes and newly diagnosed T2DM. Furthermore we found for the first time that sRAGE levels do not correlate and do not show any association with beta cell function in people with prediabetes and newly diagnosed T2DM.

The global prevalence of diabetes mellitus is rapidly rising and it is generally considered that sedentary but stressful lifestyle along with unhealthy food habits and other environmental factors may be responsible for this. It has been shown 
that dietary factors may contribute to excess accumulation of AGEs in the body [16], and AGEs have been suggested to promote beta cell dysfunction $[17,18]$ and dietary restriction of AGEs has been reported to reduce the incidence of diabetes in a mouse model of autoimmune diabetes [19]. Moreover, AGEs can act through RAGE activation, and the exogenous sRAGE and other inhibitors of RAGE ligand were shown to inhibit the development of diabetes in NOD mice $[4,5]$. Recently several studies have shown a decrease in circulating concentrations of sRAGE at the time of seroconversion to autoantibody positivity in children with prediabetes before development of type 1 diabetes $[7,8]$. These authors proposed that a declining level of sRAGE with simultaneous decrease in sRAGE/AGE ratio at seroconversion may represent a failing protection of beta cells against harmful AGEs since sRAGE can bind excessive AGEs [8]. At the same time, low circulating sRAGE at baseline has recently been shown to be significantly and independently associated with future risk of T2DM, coronary heart disease, and all-cause mortality during a median of 18 years of follow-up in a communitybased population [9]. However, this latter study was criticized as previous studies had shown higher, but not lower, levels of sRAGE are independently associated with the risk of future cardiovascular disease and all-cause mortality [11, 2022]. Moreover, circulating sRAGE levels were shown to be 1,000 times lower than needed to be efficiently capturing the circulating AGEs and therefore it is unlikely that the low levels of endogenous sRAGE to counteract the detrimental effect of AGEs might be involved in the future risk of T2DM or cardiovascular disease [11,22]. Our present finding of no relationship of endogenous sRAGE with IR and beta cell function also supports this explanation.

It was previously uncertain whether sRAGE levels alter in prediabetes and whether sRAGE levels hold any relationship with the underlying core defects of diabetes during development of T2DM, which in the present study we have tried to explore. However, we are fully aware of the limited sample size and the cross-sectional nature of our study. Future studies are therefore required to prospectively and serially measure sRAGE levels in the same subjects who develop T2DM from normoglycemia through prediabetes and to compare sRAGE with the evolution of IR and beta cell dysfunction in those individuals.

In summary, we concluded that sRAGE levels do not change in prediabetes and do not show any relationship with IR and beta cell function in people with prediabetes and newly diagnosed T2DM. These findings suggest that sRAGE is unlikely to be an important predictor of insulin resistance and beta cell dysfunction during development of T2DM. However, further studies are needed to explore the dynamics of sRAGE during development of T2DM.

\section{Abbreviations}

AGE: $\quad$ Advanced glycation end products

HOMA-IR: Homeostasis model assessment of insulin resistance

HOMA-\%B: Homeostasis model assessment of beta cell function
RAGE: Receptor for advanced glycation end products

sRAGE: Soluble receptor for advanced glycation end products

T2DM: $\quad$ Type 2 diabetes mellitus

PD + DM: Prediabetes and diabetes mellitus together.

\section{Conflict of Interests}

The authors declare that they have no conflict of interests.

\section{Authors' Contribution}

Subrata Kumar Biswas conceived and designed the study, performed experiments, analyzed and interpreted data, and drafted the paper. Sabreena Mohtarin, Sonchita Rani Mudi, and Taznuva Anwar collected data and performed experiments. Sonchita Rani Mudi, Laila Anjuman Banu, Sheikh Md. Khorshed Alam, Md. Fariduddin, and M. Iqbal Arslan interpreted data and critically revised the paper. All authors read the paper and approved the final version. Subrata Kumar Biswas is the guarantor of this work and, as such, has full access to all the data in the study and takes responsibility for the integrity of the data and the accuracy of the data analysis.

\section{Acknowledgments}

Part of this work was presented at the 50th Annual Meeting of the European Association for the Study of Diabetes, Vienna, Austria, 2014. This study was supported by a grant from the "Conversion of Bangabandhu Sheikh Mujib Medical University (BSMMU) into Center of Excellence Project, 2nd Phase" awarded to Subrata Kumar Biswas. Funding for this study was provided by a research grant of the Bangabandhu Sheikh Mujib Medical University (BSMMU), Dhaka, Bangladesh.

\section{References}

[1] A. Bierhaus, P. M. Humpert, M. Morcos et al., "Understanding RAGE, the receptor for advanced glycation end products," Journal of Molecular Medicine, vol. 83, no. 11, pp. 876-886, 2005.

[2] H. Yonekura, Y. Yamamoto, S. Sakurai et al., "Novel splice variants of the receptor for advanced glycation end-products expressed in human vascular endothelial cells and pericytes, and their putative roles in diabetes-induced vascular injury," Biochemical Journal, vol. 370, no. 3, pp. 1097-1109, 2003.

[3] A. Raucci, S. Cugusi, A. Antonelli et al., "A soluble form of the receptor for advanced glycation endproducts (RAGE) is produced by proteolytic cleavage of the membrane-bound form by the sheddase a disintegrin and metalloprotease 10 (ADAM10)," The FASEB Journal, vol. 22, no. 10, pp. 3716-3727, 2008.

[4] Y. Chen, S. S. Yan, J. Colgan et al., "Blockade of late stages of autoimmune diabetes by inhibition of the receptor for advanced glycation end products," Journal of Immunology, vol. 173, no. 2, pp. 1399-1405, 2004.

[5] J. Han, J. Zhong, W. Wei et al., "Extracellular high-mobility group box 1 acts as an innate immune mediator to enhance autoimmune progression and diabetes onset in NOD mice," Diabetes, vol. 57, no. 8, pp. 2118-2127, 2008. 
[6] C. M. Sullivan, T. S. Futers, J. H. Barrett, B. I. Hudson, M. S. Freeman, and P. J. Grant, "RAGE polymorphisms and the heritability of insulin resistance: the Leeds Family Study," Diabetes and Vascular Disease Research, vol. 2, no. 1, pp. 42-44, 2005.

[7] J. M. Forbes, J. Söderlund, F. Y. T. Yap et al., "Receptor for advanced glycation end-products (RAGE) provides a link between genetic susceptibility and environmental factors in type 1 diabetes," Diabetologia, vol. 54, no. 5, pp. 1032-1042, 2011.

[8] K. M. Salonen, S. J. Ryhaenen, J. M. Forbes et al., "Decrease in circulating concentrations of soluble receptors for advanced glycation end products at the time of seroconversion to autoantibody positivity in children with prediabetes," Diabetes Care, vol. 38, no. 4, pp. 665-670, 2015.

[9] E. Selvin, M. K. Halushka, and A. M. Rawlings, "sRAGE and risk of diabetes, cardiovascular disease, and death," Diabetes, vol. 62, pp. 2116-2121, 2013.

[10] American Diabetes Association, "Diagnosis and classification of diabetes mellitus," Diabetes Care, vol. 36, supplement 1, pp. S67-S74, 2013.

[11] S. I. Yamagishi and T. Matsui, "Soluble form of a receptor for advanced glycation end products (sRAGE) as a biomarker," Frontiers in Bioscience, vol. 2, no. 4, pp. 1184-1195, 2010.

[12] S. K. Biswas, S. R. Mudi, F. H. Mollah, A. Bierhaus, and M. I. Arslan, "Serum soluble receptor for advanced glycation end products (sRAGE) is independently associated with cigarette smoking in non-diabetic healthy subjects," Diabetes and Vascular Disease Research, vol. 10, no. 4, pp. 380-382, 2013.

[13] K. M. Salonen, S. J. Ryhaenen, J. M. Forbes et al., "Circulating concentrations of soluble receptor for AGE are associated with age and AGER gene polymorphisms in children with newly diagnosed type 1 diabetes," Diabetes Care, vol. 37, pp. 1975-1981, 2014.

[14] A. Di Pino, R. Scicali, S. Calanna et al., "Cardiovascular risk profile in subjects with prediabetes and new-onset type 2 diabetes identified by $\mathrm{HbA}_{1 c}$ according to American Diabetes Association criteria," Diabetes Care, vol. 37, no. 5, pp. 1447-1453, 2014.

[15] G. Basta, A. M. Sironi, G. Lazzerini et al., "Circulating soluble receptor for advanced glycation end products is inversely associated with glycemic control and S100A12 protein," The Journal of Clinical Endocrinology \& Metabolism, vol. 91, no. 11, pp. 4628-4634, 2006.

[16] T. Henle, "Protein-bound advanced glycation endproducts (AGEs) as bioactive amino acid derivatives in foods," Amino Acids, vol. 29, no. 4, pp. 313-322, 2005.

[17] H. Kaneto, J. Fujii, T. Myint et al., "Reducing sugars trigger oxidative modification and apoptosis in pancreatic beta-cells by provoking oxidative stress through the glycation reaction," Biochemical Journal, vol. 320, no. 3, pp. 855-863, 1996.

[18] T.-A. Matsuoka, Y. Kajimoto, H. Watada et al., "Glycationdependent, reactive oxygen species-mediated suppression of the insulin gene promoter activity in HIT cells," Journal of Clinical Investigation, vol. 99, no. 1, pp. 144-150, 1997.

[19] M. Peppa, C. He, M. Hattori, R. McEvoy, F. Zheng, and H. Vlassara, "Fetal or neonatal low-glycotoxin environment prevents autoimmune diabetes in NOD mice," Diabetes, vol. 52, no. 6, pp. 1441-1448, 2003.

[20] J. W. M. Nin, A. Jorsal, I. Ferreira et al., "Higher plasma soluble receptor for advanced glycation end products (sRAGE) levels are associated with incident cardiovascular disease and allcause mortality in type 1 diabetes: a 12 -year follow-up study," Diabetes, vol. 59, no. 8, pp. 2027-2032, 2010.

[21] R. D. Semba, L. Ferrucci, K. Sun et al., "Advanced glycation end products and their circulating receptors predict cardiovascular disease mortality in older community-dwelling women," Aging Clinical and Experimental Research, vol. 21, no. 2, pp. 182-190, 2009.

[22] S.-I. Yamagishi, "Comment on: Selvin et al. sRAGE and risk of diabetes, cardiovascular disease, and death. Diabetes 2013;62:2116-2121," Diabetes, vol. 62, no. 10, article e26, 2013. 


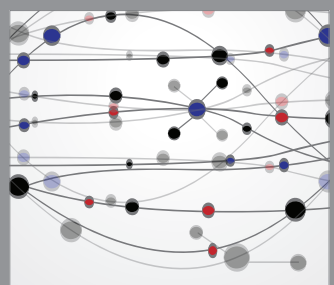

The Scientific World Journal
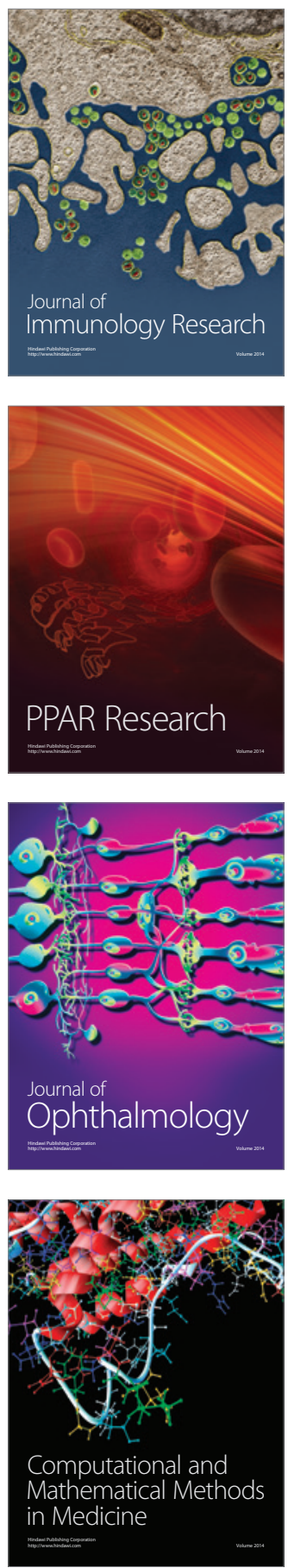

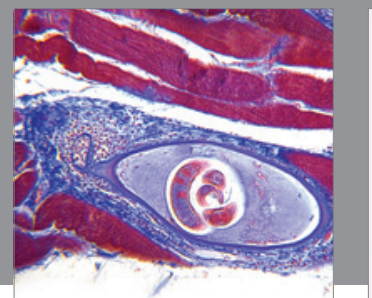

Gastroenterology

Research and Practice
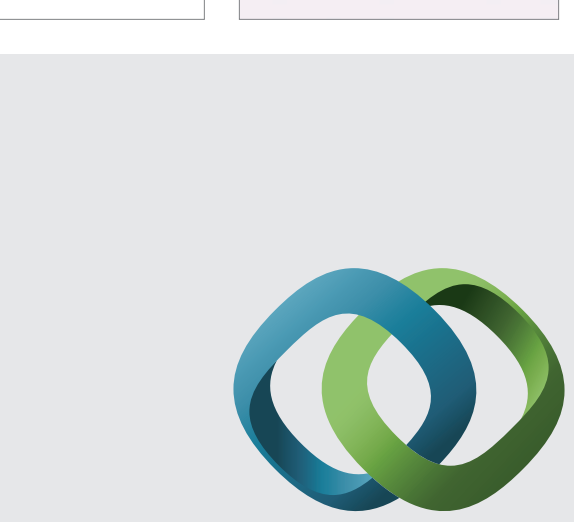

\section{Hindawi}

Submit your manuscripts at

http://www.hindawi.com
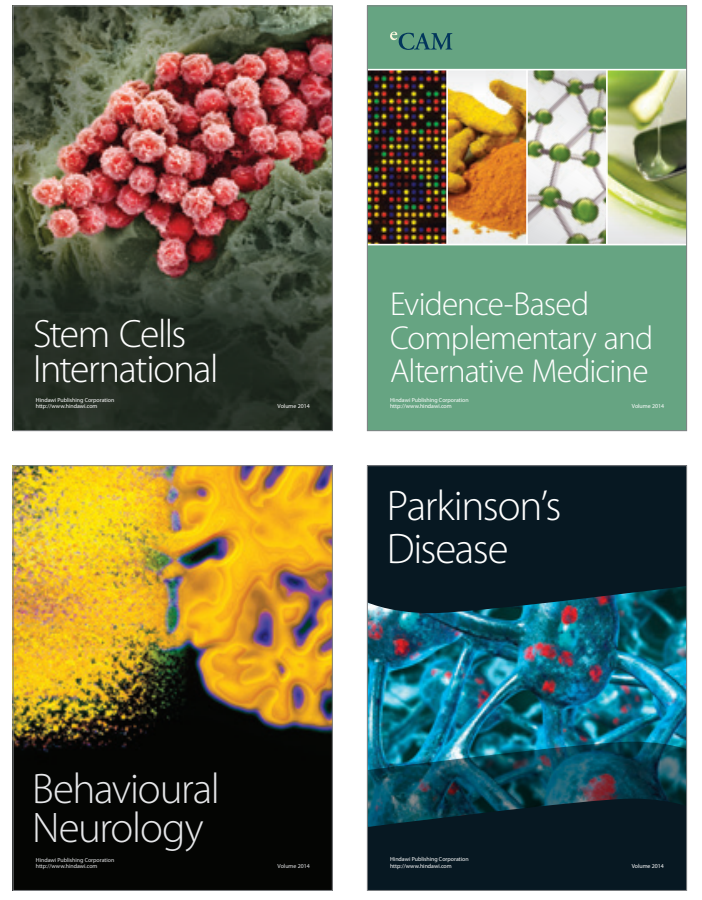
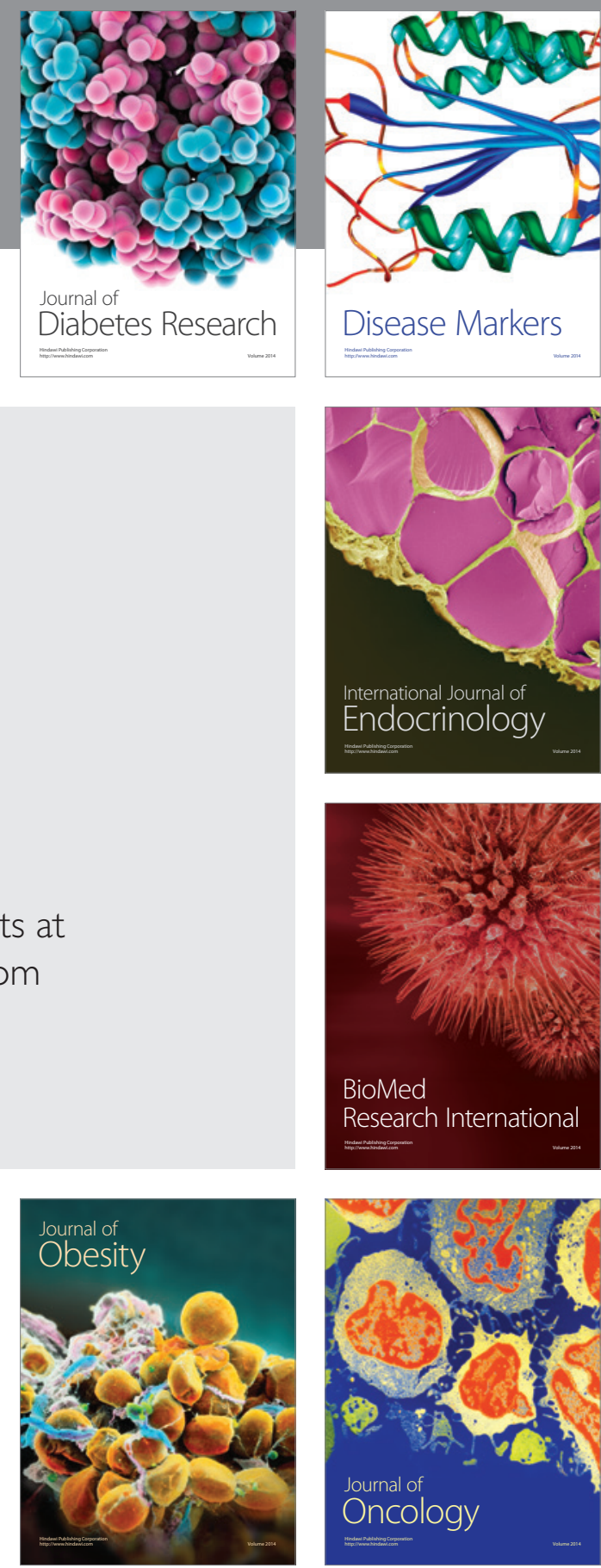

Disease Markers
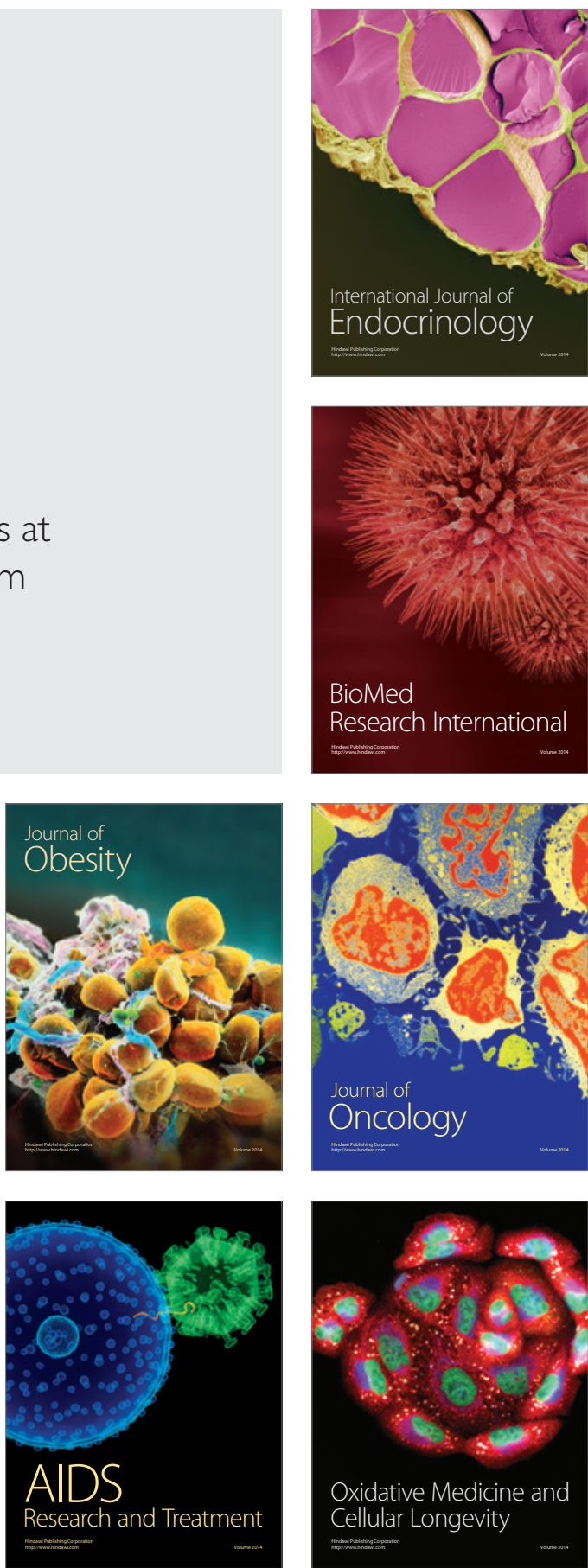